\title{
Data processing for image-based chemical sensors: unsupervised region of interest selection and background noise compensation
}

\author{
Francesca Dini • Eugenio Martinelli • \\ Roberto Paolesse • Daniel Filippini • Detlev Schild • \\ Ingemar Lundström • Corrado DI Natale
}

Received: 6 August 2011 /Revised: 16 October 2011 /Accepted: 18 October 2011 /Published online: 7 November 2011

(C) Springer-Verlag 2011

\begin{abstract}
Natural olfaction suggests that numerous replicas of small sensors can achieve large sensitivity. This concept of sensor redundancy can be exploited by use of optical chemical sensors whose use of image sensors enables the simultaneous measurement of several spatially distributed indicators. Digital image sensors split the framed scene into hundreds of thousands of pixels each corresponding to a portion of the sensing layer. The signal from each pixel can be regarded as an independent sensor, which leads to a highly redundant sensor array. Such redundancy can eventually be exploited to increase the signal-to-noise ratio. In this paper
\end{abstract}

F. Dini $\cdot$ E. Martinelli $\cdot$ C. DI Natale $(\bowtie)$

Department of Electronic Engineering,

University of Rome Tor Vergata,

Via del Politecnico 1,

00133 Roma, Italy

e-mail: dinatale@uniroma2.it

R. Paolesse

Department of Chemical Science and Technology,

University of Rome Tor Vergata,

Via della Ricerca Scientifica,

00133 Roma, Italy

\section{Filippini · I. Lundström}

Division of Applied Physics, Department of Physics,

Chemistry and Biology, Linköping University,

58183 Linköping, Sweden

\section{Schild}

Department of Neurophysiology and Cellular Biophysics, Georg-August-Universität Göttingen,

Humboldtallee 23,

37075 Göttingen, Germany

D. Schild

Bernstein Focus of Neurotechnology, University of Göttingen,

Humboldtallee 23,

37073 Göttingen, Germany we report an algorithm for reduction of the noise of pixel signals. For this purpose, the algorithm processes the output of groups of pixels whose signals share the same time behavior, as is the case for signals related to the same indicator. To define these groups of pixels, unsupervised clustering, based on classification of the indicator colors, is proposed here. This approach to signal processing is tested in experiments on the chemical sensitivity of replicas of eight indicators spotted on to a plastic substrate. Results show that the groups of pixels can be defined independently of the geometrical arrangement of the sensing spots, and substantial improvement of the signal-to-noise ratio is obtained, enabling the detection of volatile compounds at any location on the distributed sensing layer.

Keywords Optical sensors - Chemical sensors . Chemometrics/Statistics

\section{Introduction}

Observation of changes of the optical properties of sensing molecules provides a powerful method of detection of the occurrence of molecular recognition [1]. This is complemented by optoelectronics that provides a number of devices enabling assembly of sensors that transduce the changes of optical properties into electric signals. Among these devices, those based on image sensors are particularly appealing for their ability to measure arrays of different sensing layers $[2,3]$ and the distribution of analytes in dispersive media $[4,5]$.

The obvious property of image sensors is segmentation of the imaged scene into elementary units corresponding to 
the pixels of the image. Because the image sensor measures the optical properties of each pixel, each pixel may correspond to an individual sensing unit. Moreover image sensors offer a simple way of developing large sensor arrays to study the behavior of a number of replicas of the same chemical reporter [6] or even to signal the diffusion of gases into polymers [7].

Another indisputable quality of image sensors is the native connectivity to computer systems and the high quality of lowcost equipment. In this regard, sophisticated measurement can be achieved by use of a digital camera (even as simple as a webcam) as detector and a programmable light source such as a computer screen. This arrangement, known as the computer screen-photoassisted technique (CSPT), has been used to identify optical changes in biochemical assays [8] and in arrays of chemical indicators [9].

From the standpoint of detection limits, it has been demonstrated that a layer of metalloporphyrins imaged with a CSPT arrangement can detect the adsorption, in each image pixel, of femtomoles of butylamine molecules [10]. However, because image sensors enable evaluation of several replicas of similar sensors, it is possible to exploit this redundancy to improve the signal-to-noise ratio.

In this paper, an image-processing algorithm for signal enhancement for image based chemical sensor array is studied. This background noise-reduction algorithm was originally introduced to process fluorescence images of bulk stained tissues [11], a popular technique in biology used to study cell functionality, for example $\mathrm{Ca}^{2+}$ imaging in the olfactory bulb. The purpose of the algorithm is to reduce image inhomogeneity, limiting the background fluctuations in the image and increasing the signal-tonoise ratio. It exploits the information contained in the temporal dynamics of individual receptors (pixels) within a single region of interest (ROI). A ROI is defined as a subset of pixels expected to share the same signal. In a chemical sensor an ROI is a region of the image grouping receptors of the same kind.

The definition of ROIs may be trivial when the sensing layers are spotted in pre-defined locations on to the sensing layer. An alternative definition of an ROI, independent of any previous knowledge about the geometrical arrangement of the indicators, could be based on some intrinsic indicator property that can be recognized by the measurement equipment. The simplest of these properties is the color defined by the triplet of red, green, and blue intensities that the image sensor records for each pixel. Thus, if the optical indicators are sufficiently different in color the application of a clustering algorithm enables automatic segmentation of the sensing layer into ROIs each containing homogeneous, sensitive indicators [6].

In the work discussed in this paper, the properties of the background noise-reduction algorithm were applied to a layer of chemical indicators distributed on a polymer substrate and a particular neural network: the selforganizing map is used as a clustering algorithm for automatic selection of ROIs.

Results show that the background noise-reduction algorithm provides a substantial increase of the signal-tonoise ratio enabling each pixel of the image to signal the absorption of volatile compounds.

\section{Experimental}

\section{Sensing layer}

The tested chemical indicators were six metal complexes of 5,10,15,20-tetraphenylporphyrin, namely $(5,10,15,20$-tetraphenylporphyrin)palladium [PdTPP], (5,10,15,20-tetraphenylporphyrin)rhodium [RhTPP], (5,10,15,20-tetraphenylporphyrin) zinc [ZnTPP], (5,10,15,20-tetraphenylporphyrin)molybdenum [MoTPP], (5,10,15,20-tetraphenylporphyrin)iron [FeTPP], and (5,10,15,20-tetraphenylporphyrin)manganese [MnTPP], a silicon(phthalocyanine) [SiPC], and a $\mathrm{pH}$ indicator known as Nile blue (NB). The sensing molecules were dispersed in a membrane based on plasticized poly(vinyl chloride) (PVC) (membrane composition ( $w / w$ ): 33\% PVC, 66\% bisethylhexyl sebacate, and $1 \%$ indicator).

The sensing layer was prepared on a $25 \mathrm{~mm}$ diameter transparent substrate, a Thermanox plastic coverslip provided by Nunc. The polymer membrane was first cast on to the coverslip to fully coat the surface. Each indicator-polymer membrane was then spotted in several replicas above this polymeric coating, to furnish the appearance shown in Fig. 1a. The main function of the polymer layer is to support the sensing molecules while avoiding the formation of clusters and maintaining the molecules under semi-solvent conditions.

\section{Measurement setup}

The absorbance properties of the sensing spots were measured by use of the computer screen-photoassisted technique (CSPT) in which a computer screen is used as light source and a digital camera is used as detector (Fig. 1). The experiments were carried out with a Philips 170S4 computer screen and a Philips SPC650NC/97 webcam. The camera was operated at a resolution of $160 \times 120$ pixels, the signal intensities were given in camera units according to the eight-bit analog-to-digital conversion of the light intensity embedded in the camera driver.

The plastic substrate was placed in a sealed cell with transparent windows in order to be probed by light during exposure to vapors. The measurement cell was filled with organic vapors diluted in a nitrogen flow. The tested volatile organic compounds were two alcohols (ethanol 

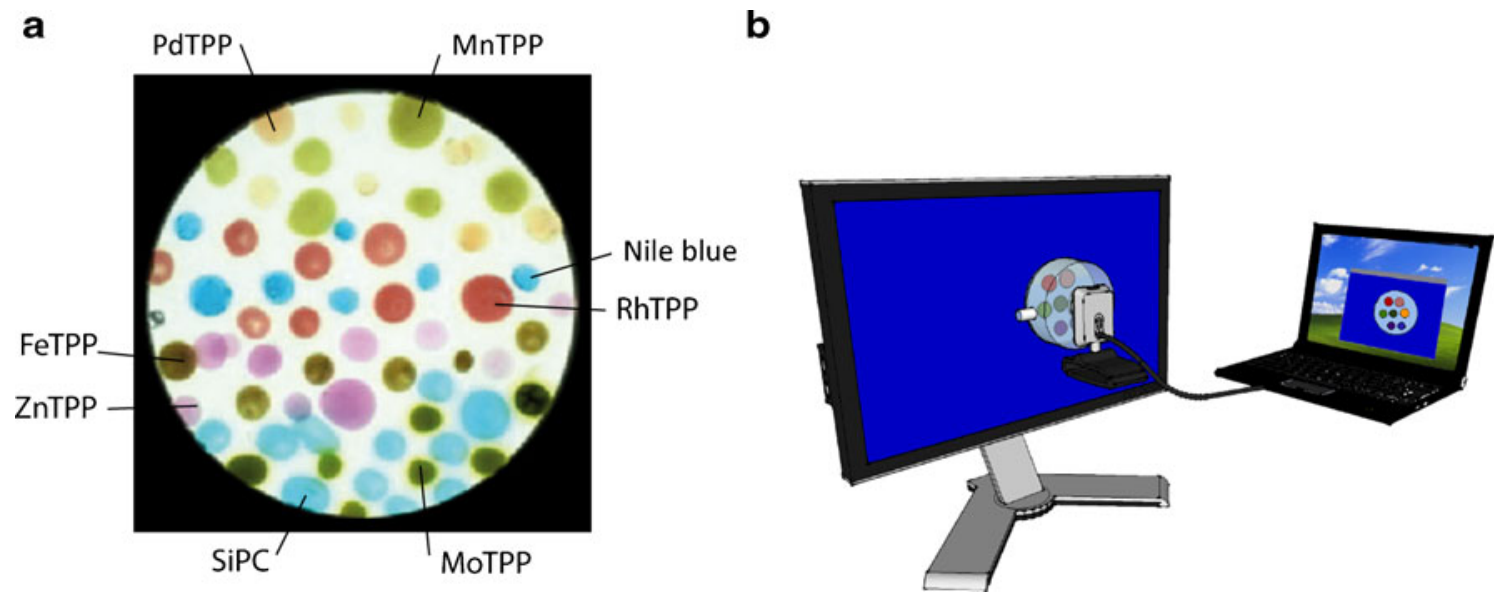

Fig. 1 (a) Picture of the sensing substrate as acquired by the webcam under white light illumination. In the figure the chemical indicators are shown. (b) Schematic diagram of the experimental setup

and butanol) and three amines (trimethylamine, triethylamine, and butylamine). Dilution factors were controlled and kept constant during each measurement by use of mass flow controllers (MKS).

It is important to remark that in the spirit of CSPT manifold illuminations are used to capture the maximum possible signal from each indicator. In this paper, for illustrative purposes, CSPT was operated with a single illumination, and green illumination was chosen because it enables measurable changes in all the indicators considered. When the sensing layer is illuminated with light of different colors, the data processing illustrated here can be conveniently used to treat the signals collected under illumination of each color.

The sensing layer was therefore illuminated with pure

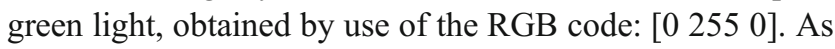
a consequence only the green channel of the camera was considered as the output signal. Camera images were sampled every $5 \mathrm{~s}$.

In accordance with the camera resolution approximately 7,500 pixels were included in the sensing area, and considering the geometrical arrangement of the measurement setup, each pixel corresponded to an area of $0.04 \mathrm{~mm}^{2}$. For each measurement, the time signals of all pixels in the sensing area were extrapolated from the raw videos and the signals of pixels within an ROI were processed with the background noise-reduction algorithm.

For the purpose of automatic definition of ROIs the sensing layer was illuminated by the computer screen programmed to display a rainbow of 50 colors from purple to red. The sequence was designed to provide a gradual mix between the three fundamental colors [9]. The layer was imaged by the webcam encoding the color in an eight-bit scale separated into the red, green, and blue channels. The signals from the red, green, and blue channels were concatenated, in this order, to form a fingerprint vector. As a result, each pixel color was encoded in a fingerprint vector composed of 50 illumination colors $\times 3$ camera color channels.

Figure 2 shows the fingerprints collected. In the ideal case of perfectly homogeneous distribution of the indicators and uniform screen illumination, only nine different fingerprints should be visible in Fig. 2a. Actually, the fingerprints are almost continuously distributed, evidence of a high level of inhomogeneity in formation of the sensing layers. This behavior becomes apparent on analysis of the data by principal-components analysis. Figure $2 \mathrm{~b}$ shows the fingerprints of the pixels projected in the plane of the first two principal components. Approximately $74 \%$ of the total variance of the data is explained in this plot. The tendency of fingerprints to cluster according to the indicator is apparent, but PCA does not furnish evidence of straightforward clustering of fingerprints.

CSPT measurements and data analysis were performed in Matlab using software written in-house.

\section{Self-organizing map}

The self-organizing map (SOM) is one of the most important neural networks characterized by unsupervised competitive learning [12]. The SOM processes a set of multivariate data to create correspondence mapping between the multivariate input data space and a representation space formed by an ordered bidimensional grid of neurons. Neurons are characterized by a vector in the input space. In practice, after learning each neuron is representative of a portion of the input data space. The learning algorithm maintains, on the grid of neurons, the topology of the input space. Thus, in the case investigated here, pixels with similar colors are mapped in neighboring neurons on the network grid. As a consequence of this correspondence, the SOM clusters the input data into classes that are defined by the network neurons. 
Fig. 2 CSPT fingerprints of the pixels of Fig. 1 (a), and scores plot of the first two principal components of the fingerprints matrix (b). Pixels are labeled according to the related indicators in accordance with the legend: 1, RhTPP; 2,

ZnTPP; 3, PdTPP; 4, Nile blue; 5, SiPC; 6, MnTPP; 7, FeTPP; 8, MoTPP; 9, background

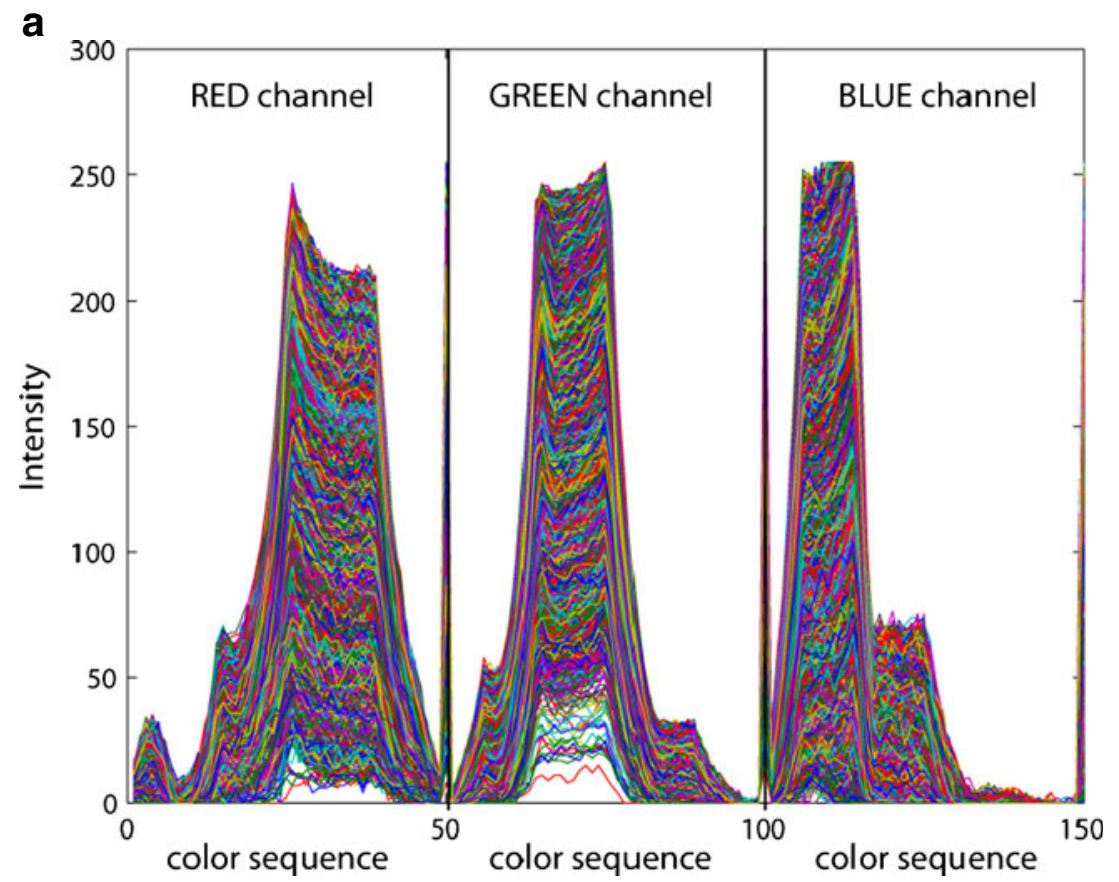

b

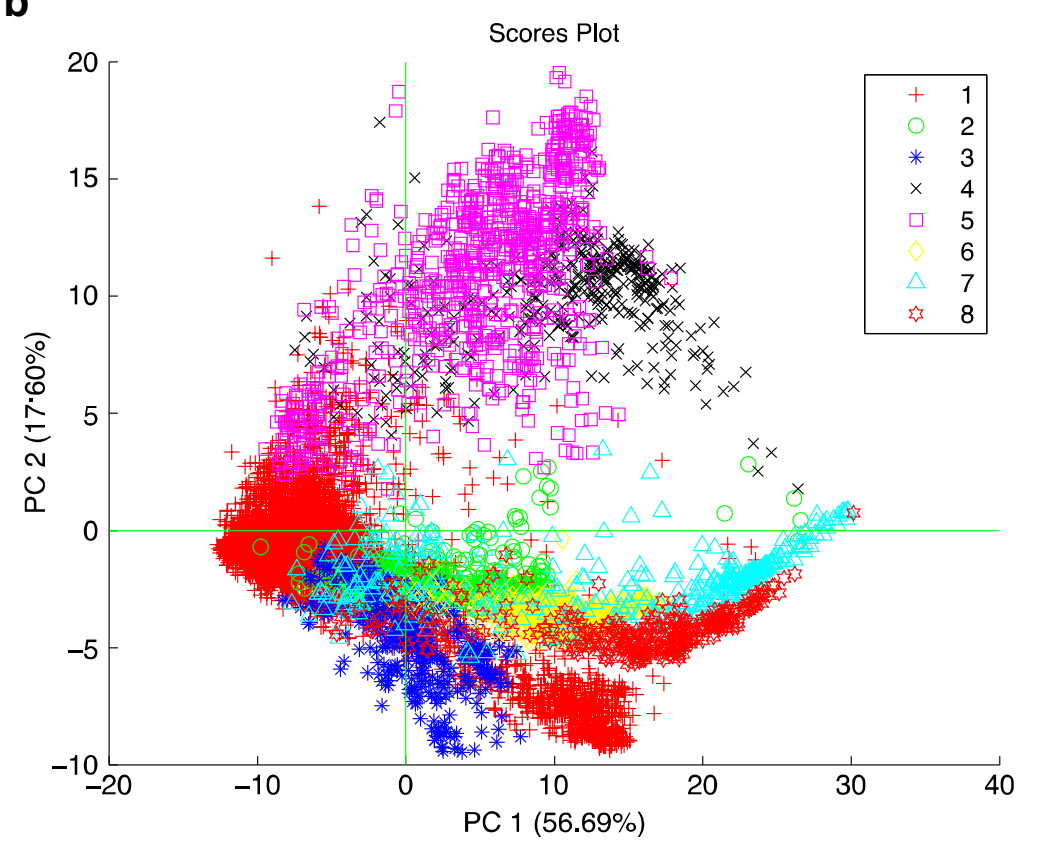

The SOM has previously been used in chemistry, for instance, to study the properties of organic compounds [13] and for classification of water pollution [14], and in color analysis to cluster the spectra of natural colors [15] and to study the color categories in color spectra databases [16].

The SOM algorithm was implemented in Matlab using the SOM Toolbox 2.0 developed at and distributed by the Laboratory of Computer and Information Science of
Helsinki University of Technology (http://www.cis.hut.fi/ somtoolbox).

\section{Background-noise-reduction algorithm}

The background-noise-reduction algorithm processes the information contained in the temporal dynamics of the signal from pixels belonging to the same region of interest 


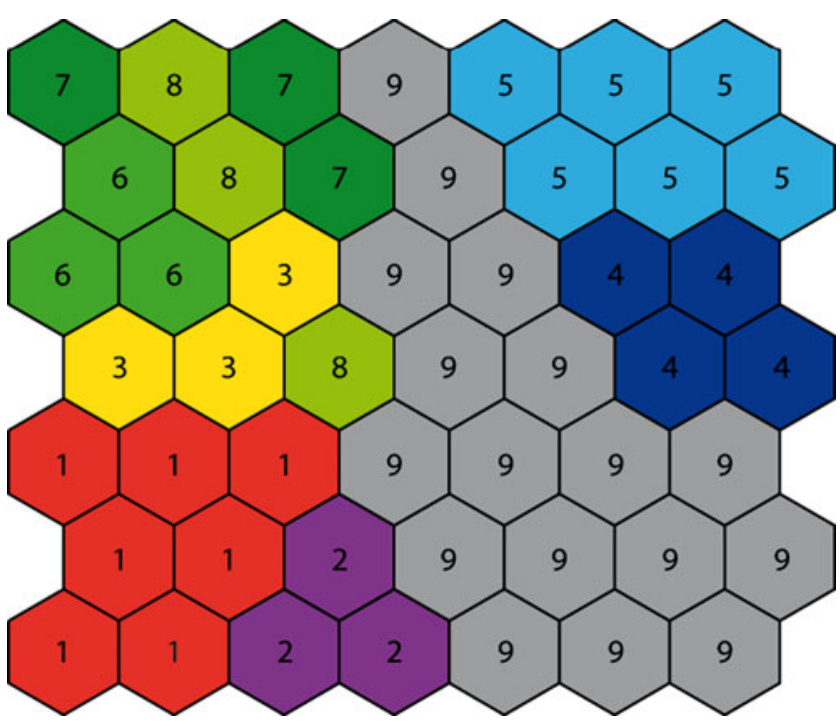

Fig. 3 SOM neurons are labeled in accordance with the indicator corresponding to the substrate portion imaged by the camera pixels. Neuron colors correspond to the actual indicator color shown in Fig. 1a; gray color indicates the uncoated area. Labels: 1, RhTPP; 2, ZnTPP; 3, PdTPP; 4, Nile blue; 5, SiPC; 6, MnTPP; 7, FeTPP; 8, MoTPP; 9, background

(ROI). An ROI is a group of pixels defining a portion of the image containing the same kind of chemical indicators. The algorithm has been thoroughly presented in a previous paper where it was introduced as a method to improve fluorescence imaging of bulk stained tissues [11].

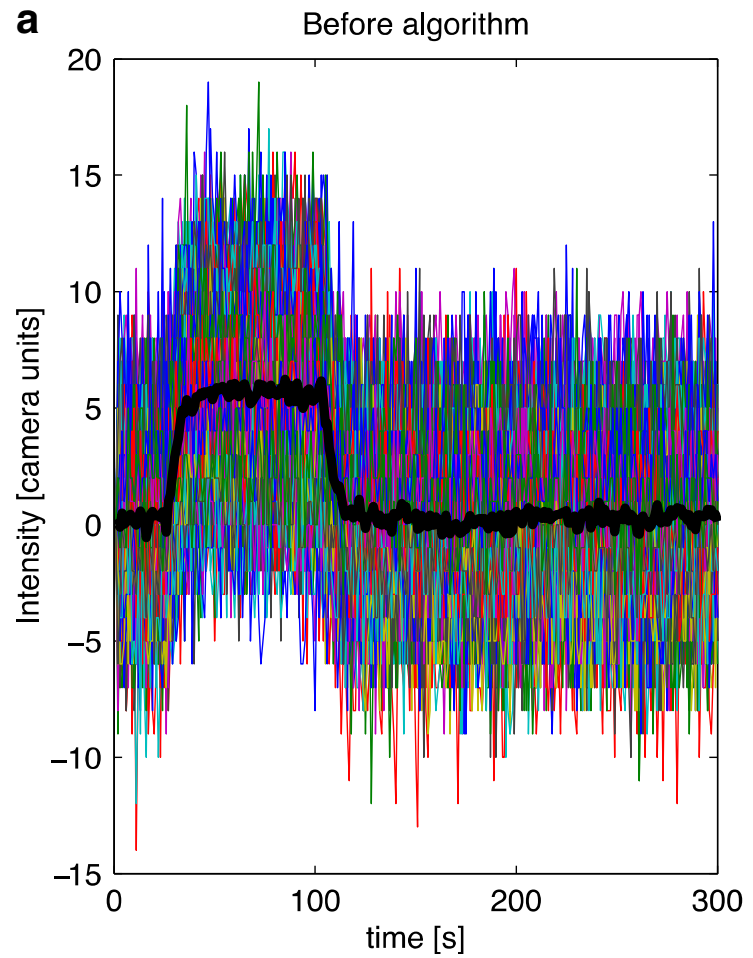

The basic assumption of the algorithm is that the measured intensity $y_{i}(t)$ of the $i$ th pixel can be expressed as the sum of three contributions:

$y_{i}(t)=u_{i} \cdot f(t)+b+n_{i}(t)$

where $b$ is the background illumination, $n_{i}(t)$ is the noise, and $f(t)$ is the supposedly common signal for all the pixels of the ROI and arises because of the chemical interaction between the indicator and volatile compound. Finally $u_{i}$ is a scaling factor of the $i$ th pixel taking into account the inhomogeneity of indicator distribution, background illumination, and detector responsivity. The quantity $f(t)$ is the unknown signal to be estimated.

In practice, the purpose of this algorithm is to retrieve the average indicator signal irrespective of its position and of the actual amount of indicator molecules.

Equation (1) can be split into time-average and timevarying parts, reducing the estimation to two separate problems.

$\bar{y}_{i}=u_{i} \cdot \bar{f}+b+n_{i}$

$\widetilde{y}_{i}=u_{i} \cdot \widetilde{f}_{i}(t)+n_{i}(t)$

The scaling factor $u_{i}$ and the signal $\tilde{f}_{i}(t)$ can then be estimated by applying the maximum likelihood method to

b

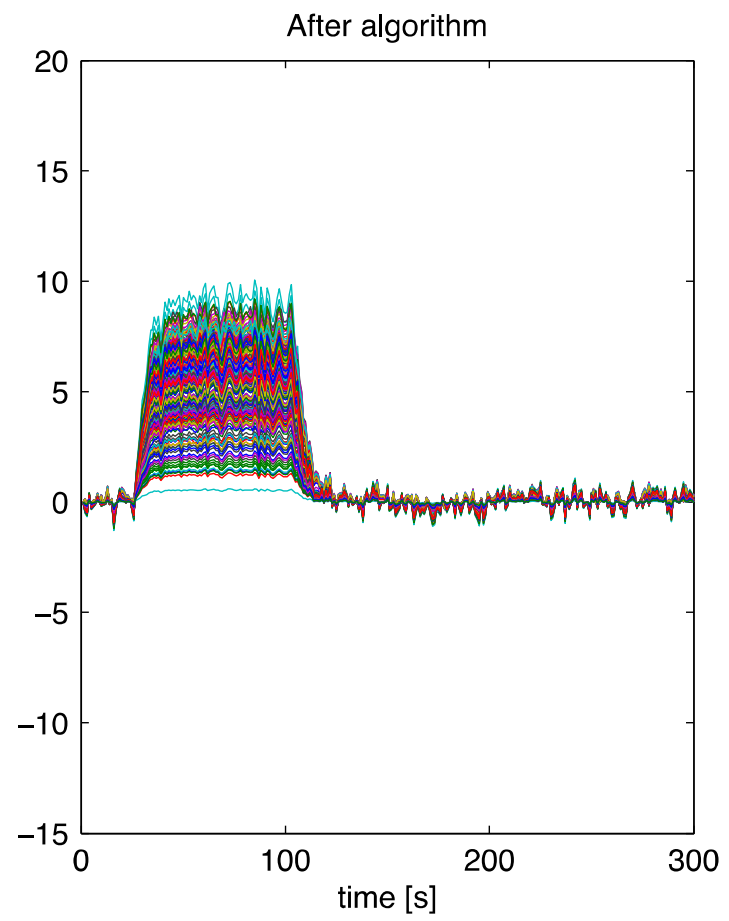

Fig. 4 Results from application of the noise-reduction algorithm to an ROI containing 219 pixels corresponding to the ZnTPP indicator: (a) raw data with their average signal shown in black; (b) the filtered signals 
the varying part of Eq. (3). Consequently $b$ and $\bar{f}$ are calculated from linear fitting of $\left(\bar{y}_{i}, u_{i}\right)$ pairs as the slope $(\bar{f})$ and the intercept $(b)$ of the regression line.

The filtered signal is eventually calculated by use of the equation:

$F_{i}(t)-u_{i} \cdot(\widetilde{f}(t)+\bar{f})$

The algorithm was implemented in Matlab.

\section{Results and discussion}

ROIs are defined as portions of the image that contain the same sensitive molecules. Use of colored optical indicators provides the opportunity for automatic assignment of ROIs. On the other hand, classification of colors is a typical CSPT application that has been shown since the beginning of the practice of this experimental technique $[17,18]$.

The collection of pixel fingerprints shown in Fig. 2 was clustered with a self-organizing map (SOM). Before being used to train the SOM, the matrix of fingerprints was linearly normalized. For each pixel, the intensity recorded for each illuminating color was divided by the sum of the intensities

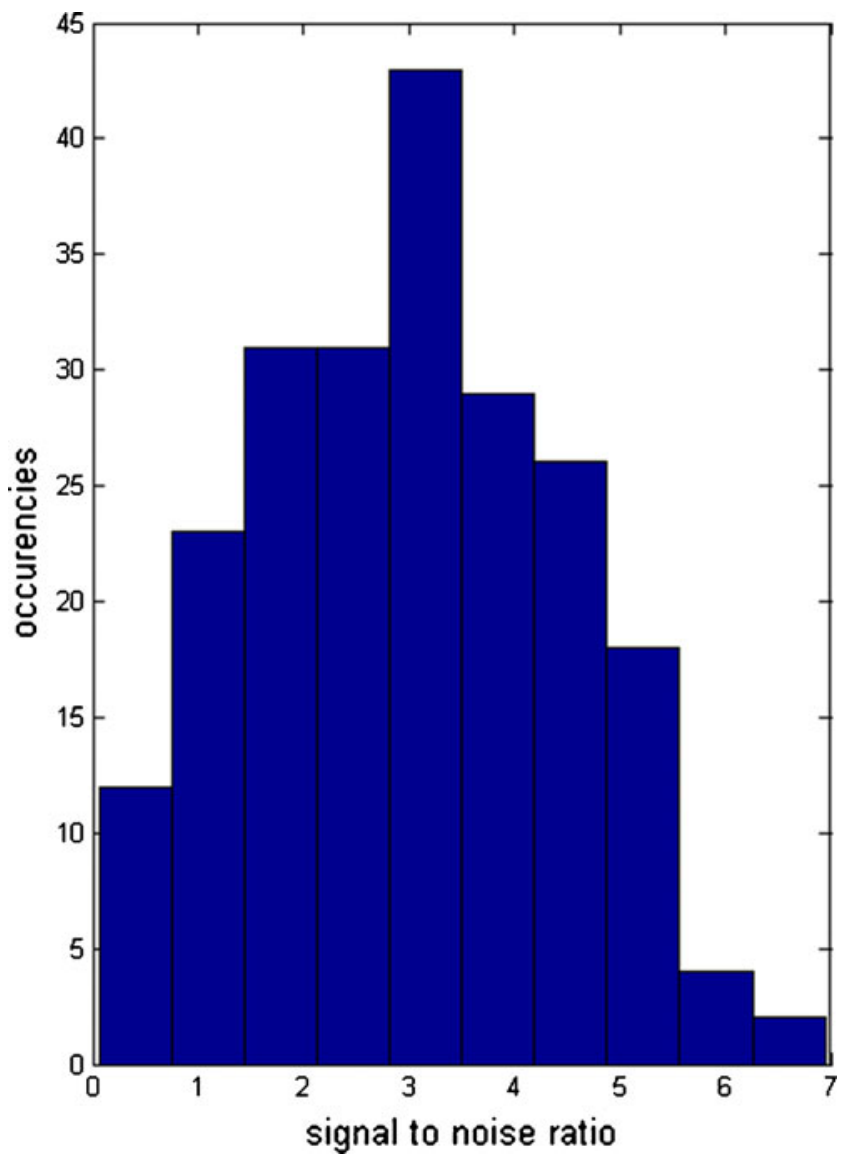

Fig. 5 Distribution of the signal-to-noise ratios of the signals of Fig. 4a of the same pixel for all the fifty colors of the sequence. This normalization was demonstrated to reduce the effect of quantitative information in sensor arrays increasing the qualitative classification of samples [19]. In this case it is expected to reduce the contribution to the measured color of the dye indicator and it is intended to remove the contribution of dye concentration to the measured dyes color.

$\bar{I}_{i j}=\frac{I_{i j}}{\sum_{j} I_{i j}}$

The training establishes a correspondence between portions of the image and the neurons of the network. In practice, each neuron of the SOM defines an ROI of the image.

The adopted definition of ROIs requires that all the pixels inside the same ROI are related to a unique indicator. In order to ensure this condition, and considering the large scatter in the fingerprints distribution shown in Fig. 2b, it is necessary to use a SOM with a number of neurons larger than the number of indicators. The optimum compromise that minimizes both the number of network units and the classification errors was achieved by use of a $7 \times 7$ neurons network arranged in hexagonal topology. The whole sensing layer was eventually portioned in 49 ROIs. As shown in Fig. 1, a large portion of the image is not occupied by the sensitive indicators, and then only $32 \mathrm{SOM}$ neurons pertain to chemically sensitive ROIs. Figure 3 shows the SOM network grid and the assignment of each neuron to the sensitive indicators and the uncoated area. Classification errors are limited to the pixels lying at the border of the spots where the color of the dye is merged with the background. However, numerically these pixels are of little importance and the errors of attribution do not substantially affect the analysis.

The background and noise-reduction algorithm was applied to the pixels of each ROI with the objective of estimating the scaling factor characteristic of each pixel and extrapolating the common signal is supposed to depend exclusively on the optical properties of the color indicator.

An illustration of the effects of the background and of noise reduction is shown in Fig. 4. Figure 4a shows the signals of the green channel of the camera from the 219 pixels of one of the three ROIs related to the indicator ZnTPP exposed to $6,000 \mathrm{ppm}$ of saturated vapor of ethanol dissolved in a pure $\mathrm{N}_{2}$ stream. During the exposure the layer was constantly illuminated with the pure green light of the screen at maximum intensity. The signals were calculated by subtracting the camera channel intensity recorded before injection of ethanol; in this way, only changes consequent to adsorption of ethanol molecules were taken into consideration. These signals correspond to the quantity $y_{i}(t)$ of Eq. (1). 


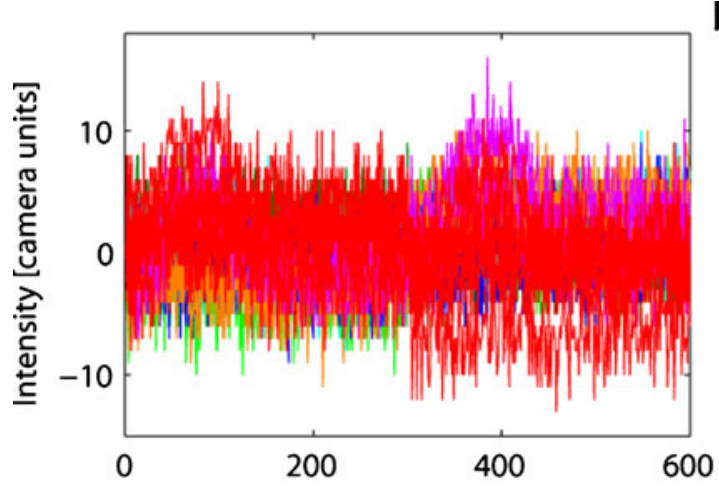

butanol

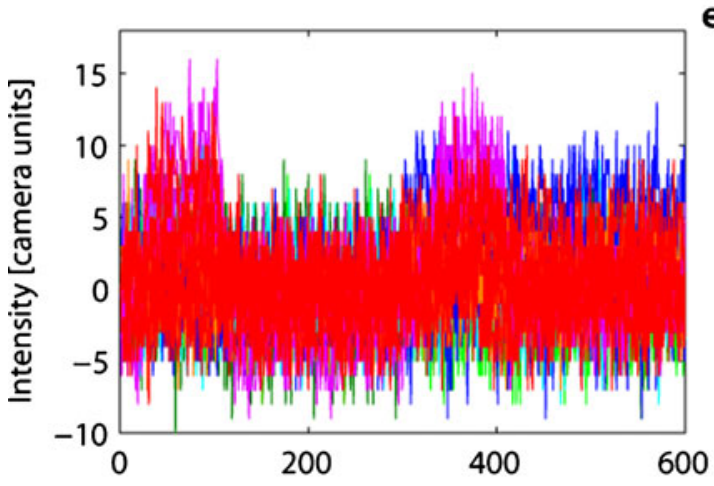

ethanol
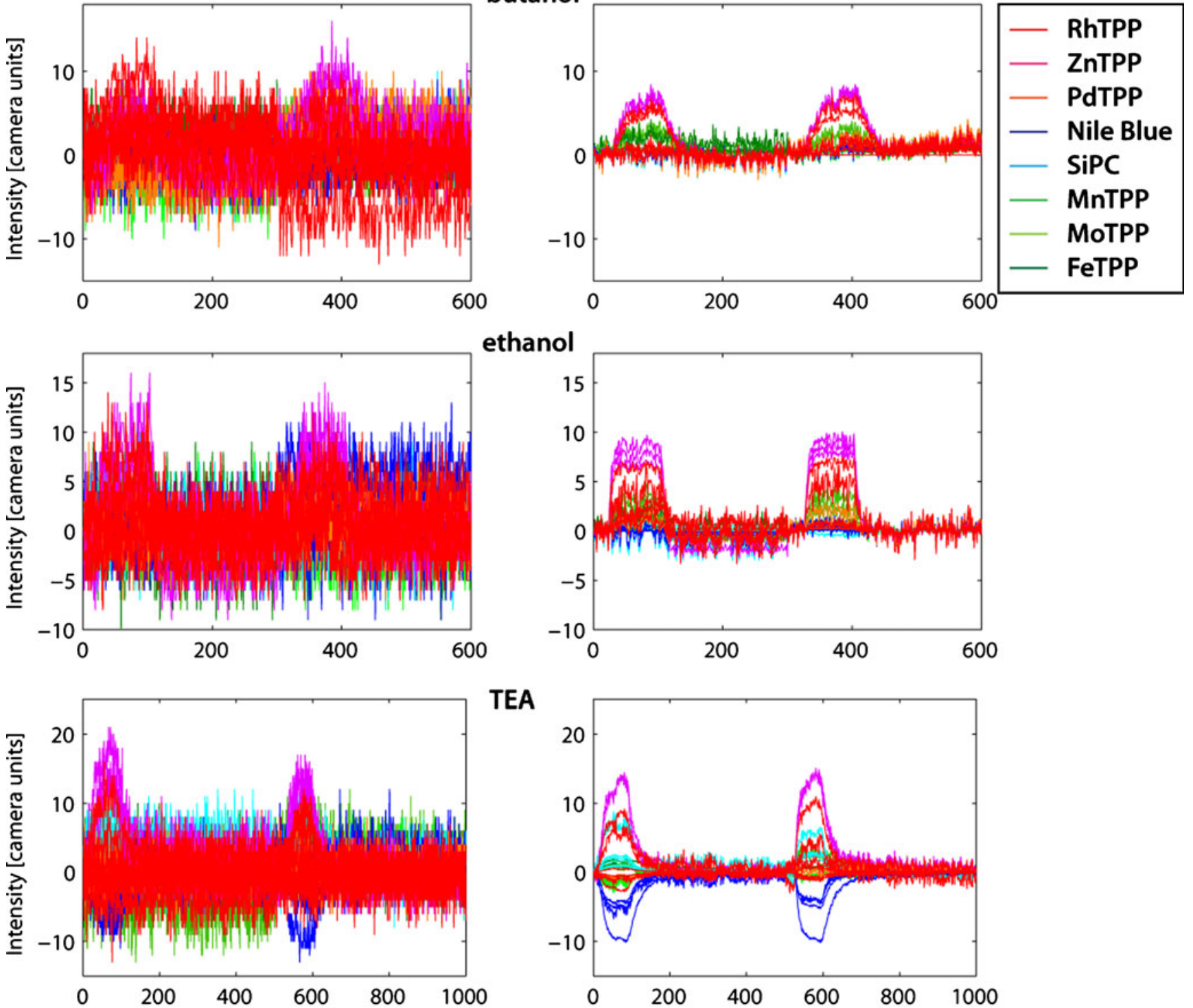

TEA

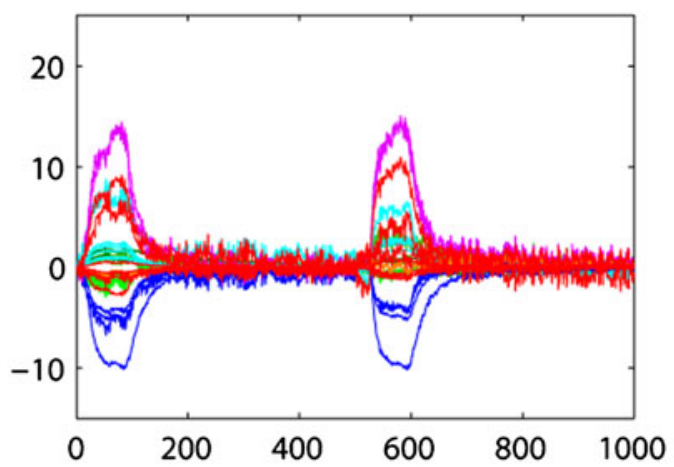

TMA
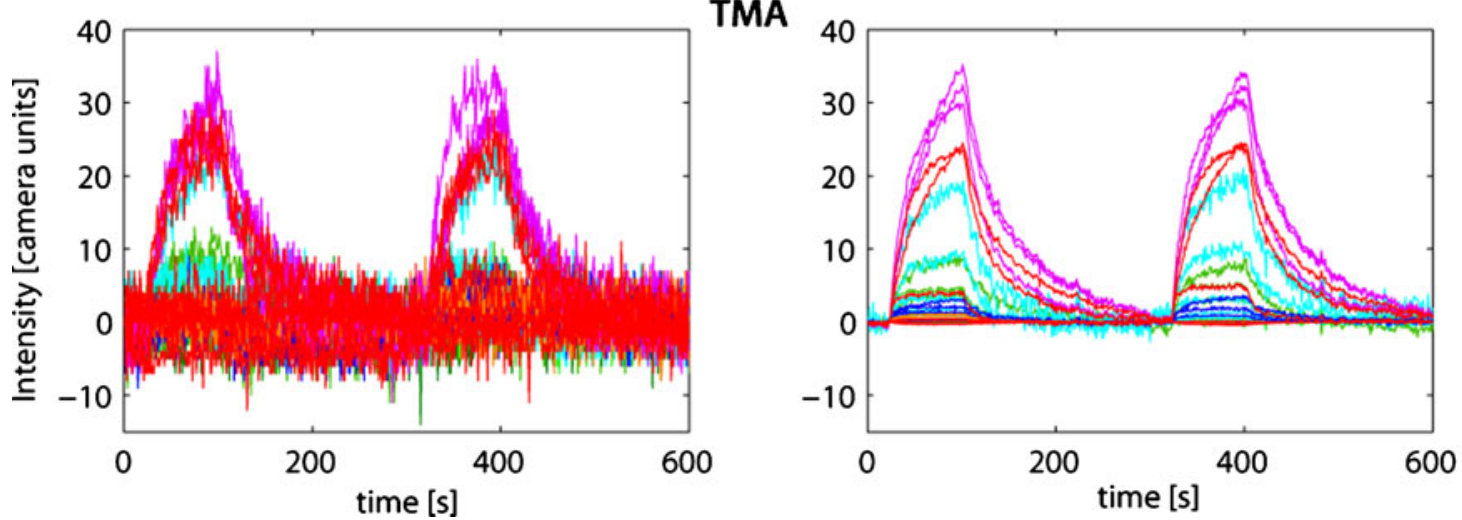

Fig. 6 Raw and processed signals from one representative pixel per class. For the significance of the colors and numbers, refer to the caption to Fig. 3

Most of the pixel signals are almost completely hidden by the fluctuations, making it difficult to appreciate the response consequent to exposure to ethanol vapor. The signals processed by the algorithm are shown in Fig. $4 \mathrm{~b}$. Although affected by evident fluctuation of residuals, the change of intensity due to exposure to the vapor can be 
a

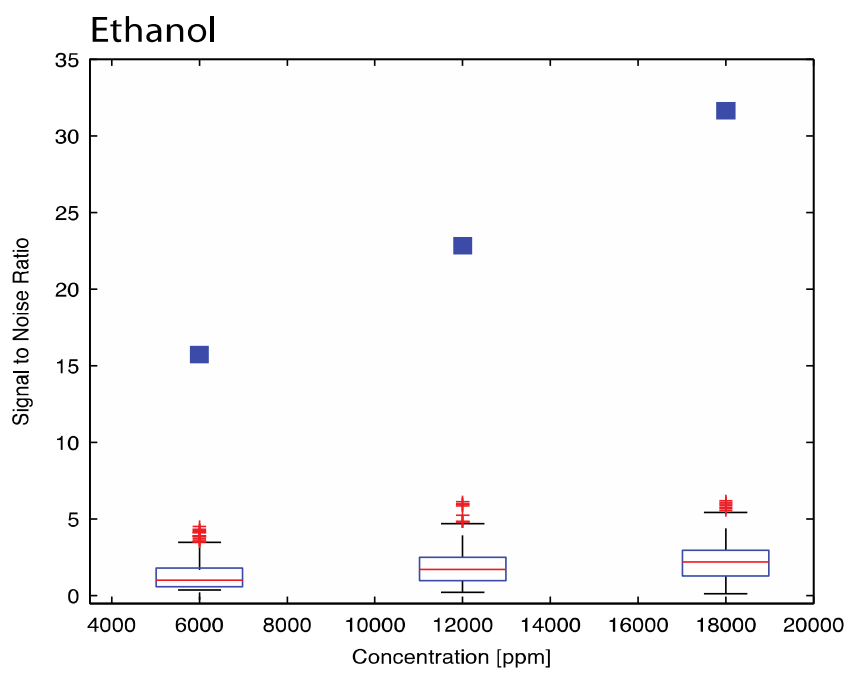

C

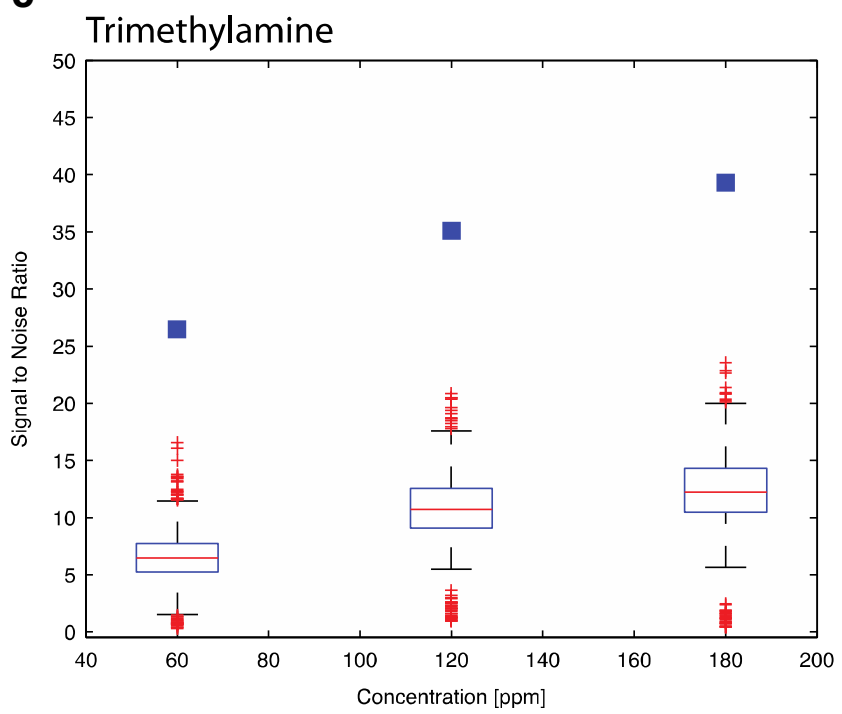

b

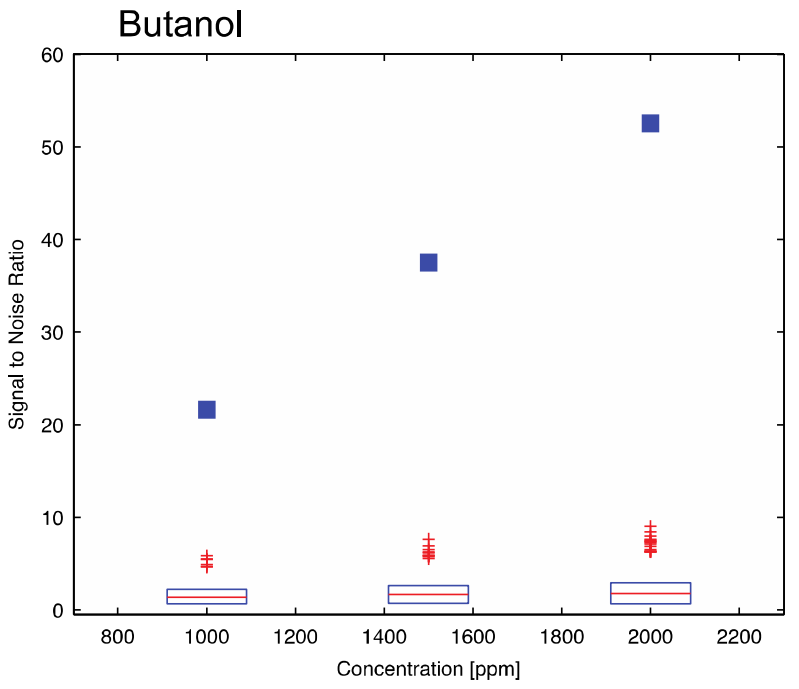

d

Triethylamine

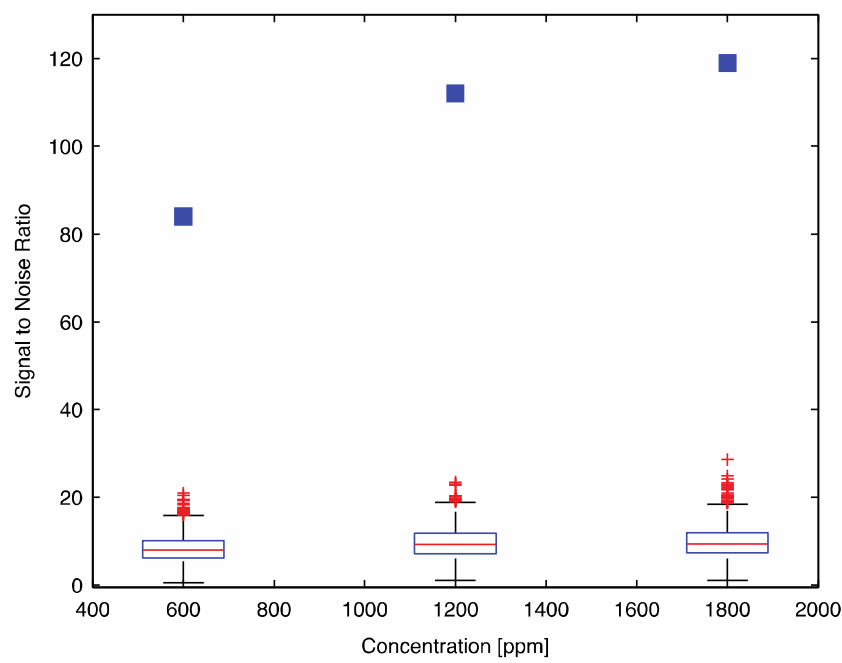

Fig. 7 Boxplot summary of the statistics. Signal-to-noise ratios for all the pixels of one of the ROIs imaging the ZnTPP indicator are plotted against the vapor concentration of two alcohols and two amines

clearly appreciated for all the pixels. It is worth remarking that the standard approach used to enhance the signal-tonoise ratio in ROI-based image analysis considers the average signal of all the pixels of the ROI. Hence, one signal replaces the signals of the pixels of the ROI. The average pixel signal is plotted in Fig. 4a. In contrast, the method discussed here improves the signal-to-noise ratio of each element of the image, preserving the individual response of the pixels.

Quantification of the efficiency of the algorithm can be achieved by comparing the signal-to-noise ratio of the pixels in the ROI before and after application of the algorithm. This quantity is calculated as the ratio between the mean and standard deviation of the signals in the range between $50 \mathrm{~s}$ and $100 \mathrm{~s}$ where, as shown in Fig. 4b, the amount of gas molecules bound to ZnTPP is in equilibrium with the concentration in the gas phase. The signal-to-noise ratio of the measured signals (Fig. 4a) is in the range 0.066.9. The signal-to-noise ratio distribution is shown in Fig. 5; approximately $45 \%$ of the pixels have a signal-tonoise ratio less than 3 whereas only $22 \%$ of the pixels have a signal-to-noise ratio larger than 4 .

The processed signal results from the product of a common signal and a constant scaling factor for each pixel, and the fluctuations still affecting the processed signals are residual common mode disturbances that are not removed by the algorithm. These residual disturbances are likely to arise because of the instability of screen luminosity and collective, pixel-independent, fluctuations of detector sensitivity. It is worthy of note that the residual fluctuations have the same 
a

Ethanol (1200 ppm)

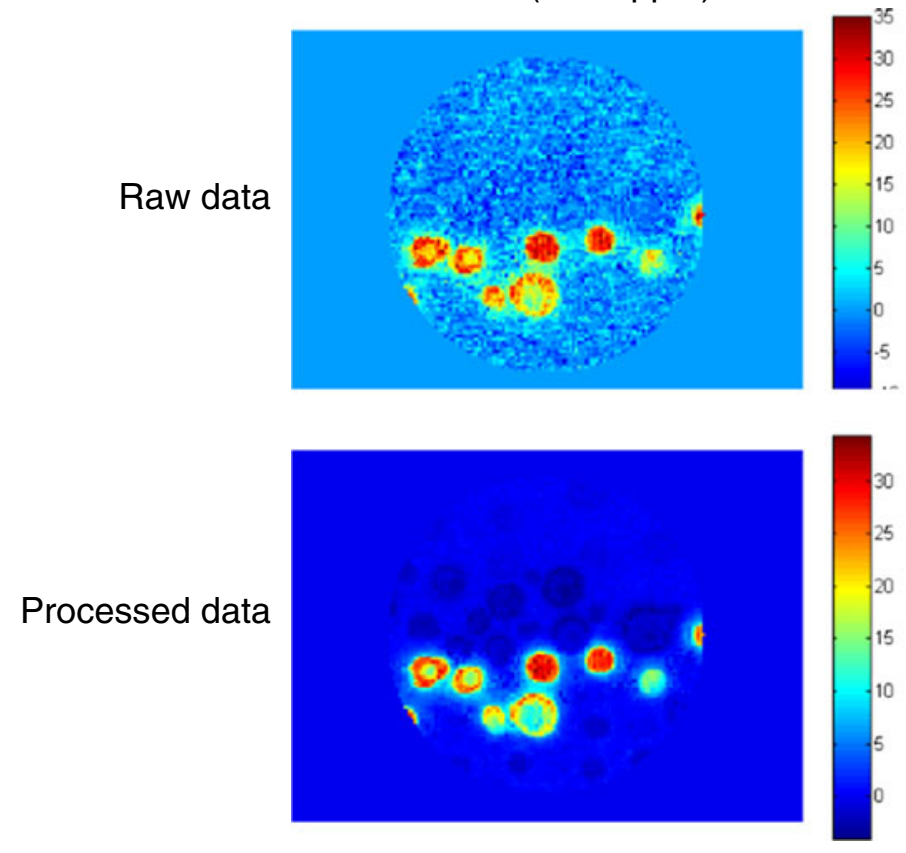

b Trimethylamine (120 ppm)
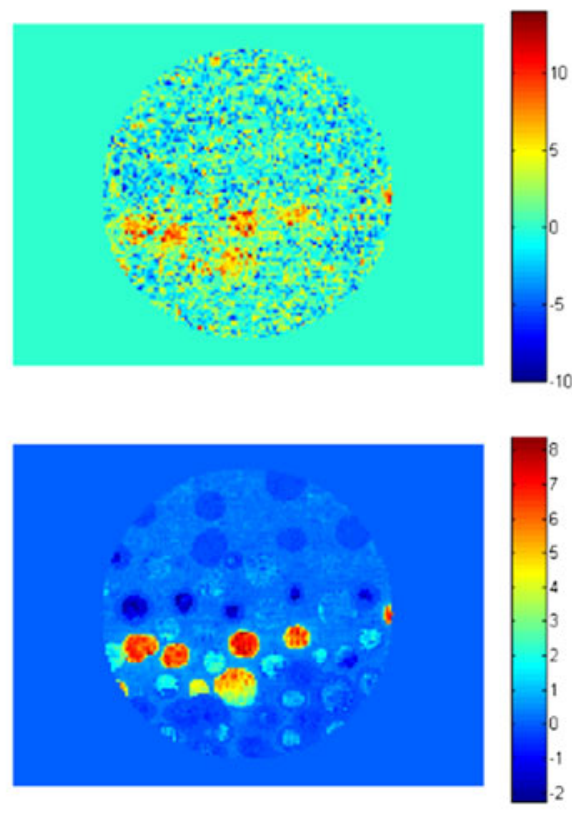

Fig. 8 Color-coded images showing the individual pixel signals during exposure to 12,000 ppm ethanol (a) and 120 ppm trimethylamine (b)

time behavior for all the pixels inside the same ROI and they are multiplied by the same scaling factor and by the useful signal. As a consequence, the processed signals inside each ROI share the same signal-to-noise ratio.

For the ROI considered in the example of Fig. $4 \mathrm{~b}$ the signal-to-noise ratio is approximately 27 , that is, approximately an order of magnitude greater than the mean signal-to-noise ratio of the unprocessed signals.

The algorithm provided similar results for the other ROIs of the image and for different volatile compounds. For illustrative purposes, the response to two consecutive exposures to four test compounds is shown in Fig. 6. For the sake of clarity the signal of only one pixel per ROI is shown here. Filtered signals show that different response patterns emerge from different ROIs, providing evidence of the recognition capabilities of the array of indicators. Figure 6 also shows the reversibility and the repeatability of the sensor signals.

To evaluate the effect of the algorithm on the response curve of the sensing units toward different volatile compounds, in Fig. 7 the signal-to-noise ratio is plotted against concentration for four volatile organic compounds (butanol, ethanol, triethylamine, and trimethylamine). The pixels belonging to one of the ROI imaging the ZnTPP indicator are considered for illustrative purpose, and the signal-to-noise ratio is represented by the boxplot for the whole ensemble of pixels signals. As suggested by Fig. 5, before application of the algorithm the raw data have a wide distribution of signal-to-noise ratio. It is worthy of note that the largest signal-to-noise ratios are achieved for a few pixels that are outliers of the statistical distribution of responses. This is likely to be because of inhomogeneous distribution of indicator molecules in the spots as a consequence of a non-optimized deposition process. Strongly interacting indicators, for example porphyrins, do, indeed, tend to aggregate during drying of the solvent giving rise to an uneven concentration of indicator in the spot.

After application of the background noise-reduction algorithm all pixels share the same signal-to-noise ratio and this value exceeds the largest signal-to-noise ratio of the unprocessed signals. The behavior of signal-to-noise ratio against concentration is compatible with the adsorption processes. Such curves can be ideally prolonged towards the unitary signal-to-noise ratio to provide a very approximate estimate of the limits of detection. Such a value is, approximately, a few ppm for both the amines, as expected considering the known affinity of ZnTPP for Lewis basis compounds.

An alternative representation of the response of the whole sensing layer to exposure to volatile compounds may be obtained by considering a color-coded image calculated by subtracting the frames acquired immediately before exposure to the gas from those captured when the sensing layer is in equilibrium with the volatile compound. In Fig. 8 the responses to exposure to $12,000 \mathrm{ppm}$ ethanol (Fig. 8a) and $120 \mathrm{ppm}$ trimethylamine (Fig. 8b) are shown. The images of filtered signals are characterized by enhanced 
contrast and a reduced noise. These figures show that the background noise-reduction algorithm leaves unaltered the possibility of tracking the spatial distribution of the response to volatile compounds.

\section{Conclusions}

In this paper we have introduced a method to enhance the signal-to-noise ratio of each pixel. The efficiency of the method is highly dependent on the unique indicator-ROI relationship. It is worthy of note, as previously mentioned, that this requirement meets one of the fundamental features of natural olfaction. ROI selection has been performed here by classifying the CSPT fingerprints of the color of pixels with a self-organizing map.

Results show that the SOM efficiently divided the pixels into classes (the SOM neurons) homogenously populated by the same kind of indicator; application of the noisereduction algorithm results in a net increase of the signalto-noise ratio of at least one order of magnitude.

Classification of the SOM is, in principle, independent of the geometrical arrangement of the sensitive indicators on the substrate, and pixels can be efficiently arranged into classes even in the extreme case of random distribution of indicators at the few hundreds of micrometers of pixel size scale.

The signal processing outlined in this paper thus makes a substantial contribution to effective use of image detectors for the development of chemical sensor arrays.

Finally, it is necessary to mention that the conjecture that pixels can be regarded as individual sensors is strictly valid for cameras where red, green, and blue colors are measured by three distinct detectors. In standard digital cameras, each pixel measures only one of the red, green, and blue colors, and the colors that are not actually measured are estimated from the neighboring pixels by use of the so-called "de-mosaicing" algorithm typically embedded in the camera processor [20]. As a consequence, pixels can be still regarded as elemental sensing units even if their independence must be carefully considered.

\section{References}

1. McDonagh C, Burke C, MacCraith B (2008) Chem Rev 108:400 422

2. Dickinson T, White J, Kauer J, Walt D (1996) Nature 382:697700

3. Ligler F, Rowe Taitt C, Shriver-lake L, Sapsford K, Shubin Y, Golden J (2003) Anal Bioanal Chem 377:469-477

4. Mustoe S, McCrossen S (2001) J Planar Chromatogr 14:252-255

5. Jaeger S, Ehni M, Eberhardt C, Rolle M, Grathwohl P, Gauglitz G (2009) Anal Bioanal Chem 395:1867-1876

6. Di Natale C, Martinelli E, Paolesse R, D'Amico A, Filippini D, Lundström I (2008) PLoS One 3:e3139

7. Dini F, Filippini D, Paolesse R, D'Amico A, Lundström I, Di Natale C (2010) Analyst 135:1245-1252

8. Filippini D, Svensson S, Lundström I (2003) Chem Commun 2:240-241

9. Filippini D, Alimelli A, Di Natale C, Paolesse R, D’Amico A, Lundström I (2006) Angew Chem Int Ed 45:3800-3803

10. Di Natale C, Santonico M, Paolesse R, Filippini D, D'Amico A, Lundström I (2010) Anal Bioanal Chem 397:613-621

11. Chen T, Lin B, Brunner E, Schild D (2006) Biophys J 90:25342547

12. Kohonen T (2001) Self-organizing maps, 3rd edn. SpringerVerlag, Berlin

13. Alonso M, Herradon B (2010) J Comput Chem 31:917-928

14. Astel A, Tsakovski S, Simeonov V, Reisenhofer E, Piselli S, Barbieri P (2008) Anal Bioanal Chem 390:1283-1292

15. K Miyazawa, J Hakkarainen, J Parkkinen, T Jaaskelainen (2005) Proceedings of the International Congress of Imaging Science, Tokyo (Japan) May 13-17, 2005

16. Raisanen E, Hauta-Kasari M (2010) Color Res Appl 35:294-303

17. Filippini D, Lundström I (2006) Anal Chim Acta 557:240-241

18. Filippini D, Lundström I (2006) J Appl Physics 99:114518114526

19. Horner G, Hierold C (1990) Sens Actuators B 2:173-184

20. K. Parulski, K. Sapulding (2003) in Digital color imaging handbook G. Sharma (editor), CRC Press, Boca Raton (FL USA) 